\title{
Colossal dielectric constant up to gigahertz at room temperature
}

Cite as: Appl. Phys. Lett. 94, 122903 (2009); https://doi.org/10.1063/1.3105993

Submitted: 13 January 2009 • Accepted: 04 March 2009 • Published Online: 23 March 2009

S. Krohns, P. Lunkenheimer, Ch. Kant, et al.

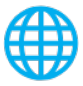

\section{ARTICLES YOU MAY BE INTERESTED IN}

Colossal dielectric constants in single-crystalline and ceramic $\mathrm{CaCu}_{3} \mathrm{Ti}_{4} \mathrm{O}_{12}$ investigated by broadband dielectric spectroscopy

Journal of Applied Physics 103, 084107 (2008); https://doi.org/10.1063/1.2902374

$\mathrm{CaCu}_{3} \mathrm{Ti}_{4} \mathrm{O}_{12}$ : One-step internal barrier layer capacitor

Applied Physics Letters 80, 2153 (2002); https://doi.org/10.1063/1.1463211

Microstructure and dielectric properties of $(\mathrm{Nb}+\mathrm{In}) \mathrm{co}$-doped rutile $\mathrm{TiO}_{2}$ ceramics

Journal of Applied Physics 116, 074105 (2014); https://doi.org/10.1063/1.4893316

\section{Challenge us.}

What are your needs for periodic signal detection?

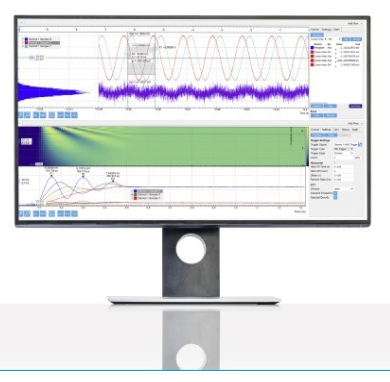




\title{
Colossal dielectric constant up to gigahertz at room temperature
}

\author{
S. Krohns, ${ }^{1}$ P. Lunkenheimer, ${ }^{1, a)}$ Ch. Kant, ${ }^{1}$ A. V. Pronin, ${ }^{2}$ H. B. Brom, ${ }^{3}$ A. A. Nugroho, ${ }^{4}$ \\ M. Diantoro, ${ }^{5}$ and A. Loidl ${ }^{1}$ \\ ${ }^{1}$ Experimental Physics V, Center for Electronic Correlations and Magnetism, University of Augsburg, 86159 \\ Augsburg, Germany \\ ${ }^{2}$ Hochfeld-Magnetlabor Dresden (HLD), Forschungszentrum Dresden-Rossendorf (FZD), 01314 Dresden, \\ Germany and A. M. Prokhorov Institute of General Physics, RAS, 119991 Moscow, Russia \\ ${ }^{3}$ Kamerlingh Onnes Laboratory, Leiden University, P.O. Box 9504, 2300 RA Leiden, The Netherlands \\ ${ }^{4}$ Department of Physics, Bandung Institute of Technology, Jl. Ganesha 10 Bandung 40132, Indonesia \\ ${ }^{5}$ Department of Physics, State University of Malang, Jl. Surabaya 6 Malang 65145, Indonesia
}

(Received 13 January 2009; accepted 4 March 2009; published online 23 March 2009)

\begin{abstract}
The applicability of recently discovered materials with extremely high ("colossal") dielectric constants, required for future electronics, suffers from the fact that their dielectric constant $\varepsilon^{\prime}$ only is huge in a limited frequency range below about $1 \mathrm{MHz}$. In the present report, we show that the dielectric properties of a charge-ordered nickelate, $\mathrm{La}_{15 / 8} \mathrm{Sr}_{1 / 8} \mathrm{NiO}_{4}$, surpass those of other materials. Especially, $\varepsilon^{\prime}$ retains its colossal magnitude of $>10000$ well into the gigahertz range. (c) 2009 American Institute of Physics. [DOI: 10.1063/1.3105993]
\end{abstract}

In recent years, large effort has been put into the development and characterization of colossal- $\varepsilon^{\prime}$ materials. Especially, the recent discovery ${ }^{1,2}$ of values of the dielectric constant $\varepsilon^{\prime}$ up to about $10^{5}$ in $\mathrm{CaCu}_{3} \mathrm{Ti}_{4} \mathrm{O}_{12}$ (CCTO) has aroused tremendous interest. In addition, there are also some reports of other colossal- $\varepsilon^{\prime}$ materials (e.g., Refs. 3-5), mainly transition metal oxides. CCTO and other materials stand out due to their colossal- $\varepsilon^{\prime}$ values being nearly temperature independent over a broad range around room temperature. But in all these materials, a strong frequency dependence is observed, showing the signature of relaxational contributions. The extensive investigations of CCTO have revealed that the observed relaxation features are of the nonintrinsic MaxwellWagner (MW) type ${ }^{6-8}$ They are due to heterogeneity of the sample, which is composed of a bulk region with relatively high conductivity and one or several insulating thin layers. These layers can arise from surface effects (e.g., depletion regions of Schottky diodes at the electrodes) or internal barriers (e.g., grain boundaries). However, this is rather irrelevant from an application point of view. Thus, although in CCTO the exact mechanism is not yet finally clarified, the interest in this material is still high. Unfortunately, at room temperature, the relaxation in CCTO leads to a decrease of $\varepsilon^{\prime}$ in the megahertz region and around gigahertz only values of the order of 100 are observed. ${ }^{6,9,10}$ In contrast, electronic applications, e.g., in computer technology or telecommunications nowadays require much higher frequencies, up to the gigahertz range.

In a search for a better material, we found that $\mathrm{La}_{15 / 8} \mathrm{Sr}_{1 / 8} \mathrm{NiO}_{4}$ (LSNO) is much more promising for fulfilling the requirements of modern electronics than CCTO. The system $\mathrm{La}_{2-x} \mathrm{Sr}_{x} \mathrm{NiO}_{4}$ attracted much interest due to the formation of charge order in large parts of its phase diagram. ${ }^{11,12}$ In the present work, we report the results of broadband dielectric measurements of single crystalline LSNO in a frequency range up to $1 \mathrm{GHz}$.

The LSNO crystals were prepared by the traveling solvent floating zone growth technique. Crystal qualities were

${ }^{a)}$ Electronic mail: peter.lunkenheimer@physik.uni-augsburg.de. examined by scanning electron microscopy, electron probe microanalysis, and x-ray Laue backscattering. The oxygen content of the crystals selected for the present study was 4.005-4.010. The crystal orientation was determined with $\mathrm{x}$-ray diffractometry: the surface plane of the crystal on which the contacts were applied was perpendicular to the $\langle 110\rangle$ direction with one of the plane edges along the $\langle 001\rangle$ direction. For the dielectric measurements, silver paint or sputtered gold contacts were applied at opposite sides of the platelike samples. Frequency-response analysis and a coaxial reflection technique were used to determine the dielectric properties. ${ }^{13}$ In the far- and midinfrared (mid-IR) range, reflectivity measurements were carried out using a Bruker Fourier-transform spectrometer IFS $113 \mathrm{v}$. More detailed experimental information can be found in Ref. 14 .

Figure 1 shows the temperature dependence of the dielectric constant and loss tangent measured with silver-paint contacts at various frequencies. In $\varepsilon^{\prime}(T)$, a strong step from low-temperature values of about 300 up to colossal values of about 17000 is revealed, shifting with the measurement frequency. Correspondingly, the loss tangent shows wellpronounced peaks. These are the typical signatures of a relaxation and the overall behavior qualitatively resembles that of CCTO (see, e.g., Ref. 2). However, already in this temperature-dependent plot, strong quantitative differences in the relaxation behavior become evident. While in CCTO the relaxation step at, e.g., $1 \mathrm{MHz}$ occurs at about $200 \mathrm{~K}$, in LSNO it is observed at a much lower temperature of about $60 \mathrm{~K}$ and, thus, the region of constant $\varepsilon^{\prime}$ is much broader. In addition to this main relaxation feature, a second smearedout relaxation step is observed in $\varepsilon^{\prime}(T)$ [Fig. 1(a)] leading to values at high temperatures and low frequencies of about 50000 . Correspondingly, broad shoulders in $\tan \delta$ show up. Interestingly, if CCTO is investigated in a sufficiently broad temperature and frequency range, also there a second relaxation is detected. ${ }^{6,9,10,15}$ It should be noted that the loss tangent of LSNO [Fig. 1(b)] is at high frequencies smaller than in CCTO. ${ }^{2}$ However, its room temperature value, arising from the second relaxation and charge transport, still needs to be reduced for a straightforward application as dielectric 


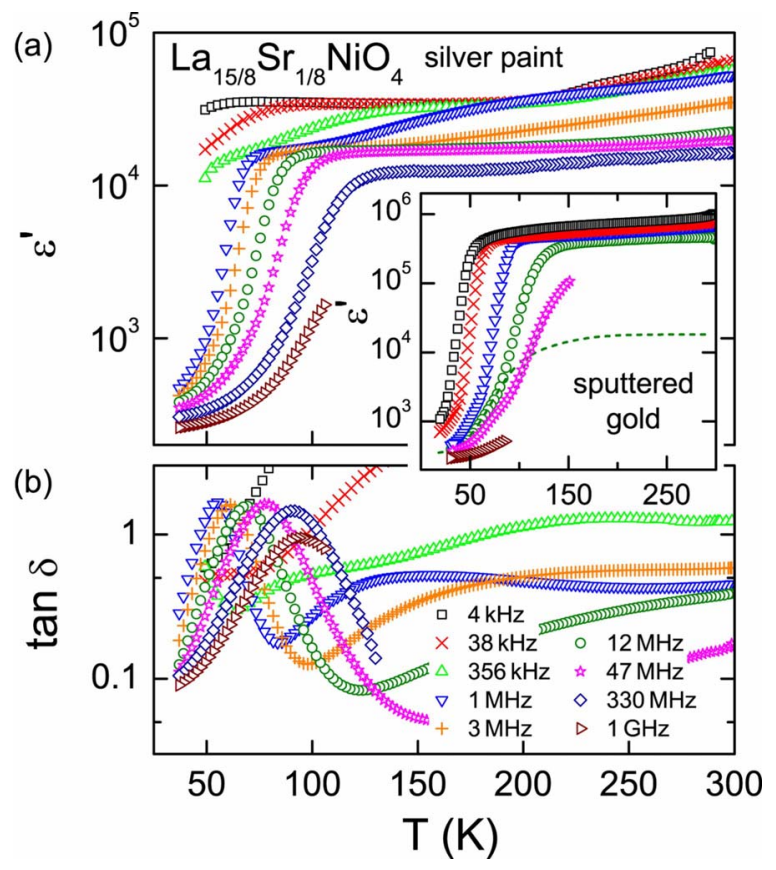

FIG. 1. (Color online) Temperature dependence of the dielectric properties of LSNO for various frequencies. The main frames show the dielectric constant (a) and loss tangent (b) measured with silver-paint contacts. The inset shows the dielectric constant obtained on the same sample with sputtered gold contacts. The dashed line represents an educated guess indicating the presence of a second relaxation. For the highest frequencies the data partly were out of the resolution limit of the instrument (not shown).

material. The inset of Fig. 1 shows the temperaturedependent dielectric constant of the same LSNO crystal, after removing the silver-paint contacts and applying sputtered gold contacts. Here, truly colossal values of $\varepsilon^{\prime}$ are found, reaching nearly $10^{6}$. Also in CCTO a strong enhancement of $\varepsilon^{\prime}$ for sputtered contacts was observed. ${ }^{6,9,10}$

The key result of the present work is revealed by the frequency-dependent plot of $\varepsilon^{\prime}$ in Fig. 2(a), extending over nine frequency decades up to $1 \mathrm{GHz}$. Quite in contrast to CCTO (Ref. 10) (see inset of Fig. 2 for a comparison), at room temperature the dielectric constant of LSNO is not affected by the main relaxation and retains its colossal magnitude up to the highest investigated frequency $(430 \mathrm{MHz})$. A measurement up to higher frequencies was not possible as there these high values are out of the resolution limit of the instrument. However, already from the fact that even for 150 $\mathrm{K}$ the relaxation step is not shifted into the frequency window, it is clear that colossal $\varepsilon^{\prime}$ values are retained even beyond $1 \mathrm{GHz}$. To obtain quantitative information, $\varepsilon^{\prime}(\nu)$ and $\sigma^{\prime}(\nu)$ [Fig. 2(b)] were fitted assuming an equivalent circuit just as used for CCTO. ${ }^{6,10}$ This description assumes a MW mechanism as origin of the colossal $\varepsilon^{\prime}$ and the two relaxations but also can be taken as a purely phenomenological description for parameterization purposes. It comprises two parallel $R C$ circuits to take into account the relaxations, connected in series to the bulk element. The latter is assumed to exhibit dc conductivity and a power-law contribution, $\sigma^{\prime}$ $\propto \nu^{s}(s<1)$, commonly termed "universal dielectric response" (UDR) (Ref. 16) and ascribed to hopping of localized charge carriers. ${ }^{17}$ The lines shown in Fig. 2 are fits with this circuit, leading to a reasonable description of the experimental data. A straightforward extrapolation of the obtained characteristic times of the main relaxation leads to a room

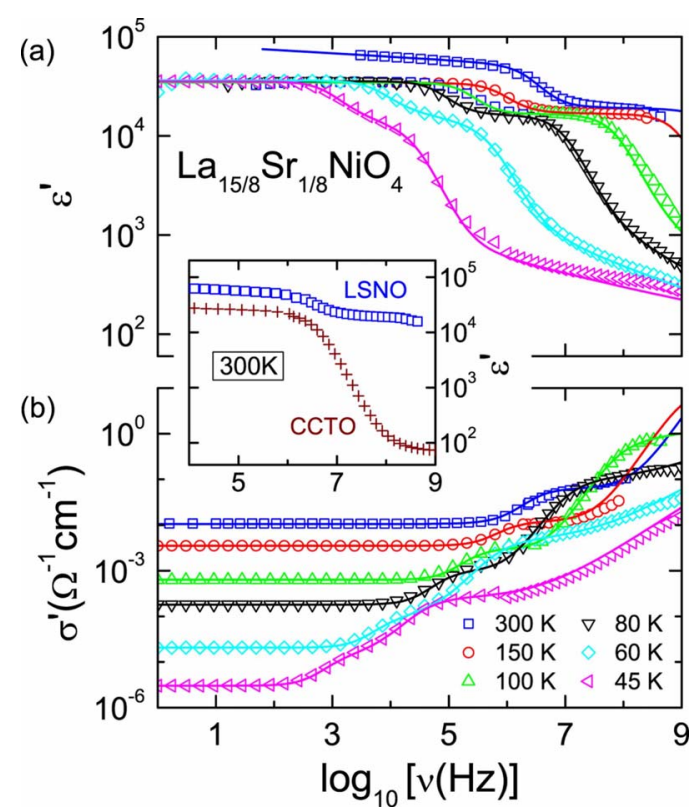

FIG. 2. (Color online) Broadband dielectric spectra of LSNO for various temperatures. (a) Dielectric constant. (b) Conductivity. The lines are fits with an equivalent circuit as used in Refs. 6 and 10. The inset shows a comparison of $\varepsilon^{\prime}(\nu)$ at room temperature in LSNO and CCTO (from Ref. 10).

temperature value of $\tau=1.3 \times 10^{-11} \mathrm{~s}$, corresponding to a frequency of $12 \mathrm{GHz}$. This again evidences that colossal $\varepsilon^{\prime}$-values can be expected well up into the gigahertz regime.

It is interesting that in Fig. 2(a), even at frequencies beyond the main relaxation (e.g., at $1 \mathrm{GHz}$ for $\nu \leq 60 \mathrm{~K}$ ), with $\varepsilon^{\prime} \approx 300$ the dielectric constant of LSNO still is exceptionally high, clearly exceeding the corresponding, already rather high value in CCTO of about 85 (inset of Fig. 2). ${ }^{2,6,10}$ It should be noted that if a MW mechanism is assumed, only here the intrinsic $\varepsilon^{\prime}$ is detected. ${ }^{8}$ To obtain information on the origin of this high value in LSNO, in Fig. 3 the dielectric results on $\varepsilon^{\prime}(\nu)$ are shown together with those from an IR measurement performed at room temperature. While a detailed analysis of the IR results are out of the scope of the present work, Fig. 3 clearly reveals that in LSNO, just as in $\mathrm{CCTO}^{2,18}$ rather strong phonon resonances exist in the terahertz region pointing to a high ionic polarizability. However, these modes lead to a "static" dielectric constant (read off at

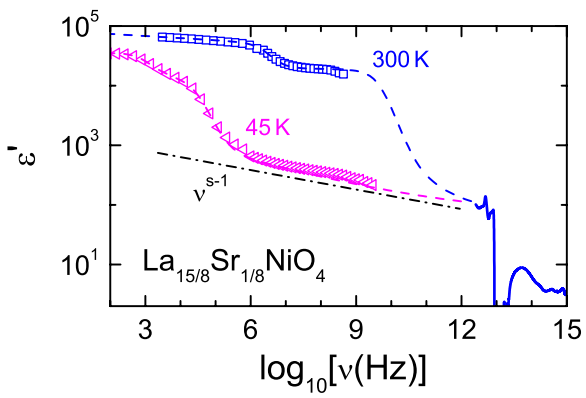

FIG. 3. (Color online) Comparison of the frequency-dependent dielectric constant of LSNO from dielectric and IR measurements. The dielectric spectra (shown as symbols) are presented for two temperatures. The IR measurements, shown as solid line, were performed at room temperature. The dashed lines are the same fits as shown in Fig. 2(a), but extrapolated up to the IR range. The dash-dotted line indicates the power-law behavior of $\varepsilon_{\text {UDR }}^{\prime}$ due to hopping conductivity. 
the lowest frequency of the IR curve) of about 90 only. While of similar magnitude as in CCTO, ${ }^{2,18}$ this cannot explain the observed values of 300 at $1 \mathrm{GHz}$ and $45 \mathrm{~K}$ in LSNO. A solution for this apparent discrepancy is provided by the UDR, which via the Kramers-Kronig relation leads to a contribution $\varepsilon_{\mathrm{UDR}}^{\prime} \propto \nu^{s-1}$, with $s<1$, as indicated by the dash-dotted line in Fig. 3. ${ }^{16}$ The dashed lines shown in Fig. 3 correspond to the fit curves of Fig. 2(a), which were adjusted to match the IR results. Obviously, in LSNO the UDR contribution is stronger than in CCTO and plays an important role in the generation of a high $\varepsilon^{\prime}$ at frequencies beyond the relaxation. When considering Fig. 3, one should be aware that the electric field directions were not identical in the dielectric $(E \|\langle 110\rangle)$ and IR experiment $(E \perp\langle 110\rangle)$. However, within the scale of Fig. 3, only a small influence of the field direction on the strength of the phonon modes can be expected.

Concerning the origin of the colossal $\varepsilon^{\prime}$ and the observed relaxations in LSNO, the performed measurements with silver paint and sputtered contacts demonstrate that surface effects must play some role. Thus, part of the observed phenomena can be explained in terms of extrinsic MW effects due to contacts (Schottky diodes) or more complex surface effects. ${ }^{6,8-10}$ Grain boundaries can be excluded as in the present work single crystalline samples were investigated. However, it should be noted that in the measurements with silver-paint contacts, two relaxations are clearly detected (Figs. 1 and 2) and, thus, a second mechanism enhancing $\varepsilon^{\prime}$ must be present. In addition, a close inspection of the curves obtained with sputtered gold contacts (inset of Fig. 1) reveals a shoulder at low temperatures, and thus they are composed of two separate contributions too. The first, low-temperature one may well lead to values of the order of $10^{4}$ (dashed line in the inset of Fig. 1) just as the main relaxation for the silver-paint electrodes [Fig. 1(a)]. Thus, only the second, high-temperature relaxation seems to differ between silverpaint and sputtered contacts and, thus, can be assumed to be due to surface effects.

In contrast, for the first relaxation, different origins should be considered: In Refs. 4 and 5, a high $\varepsilon^{\prime}$-magnitude and relaxational behavior, found in $\mathrm{La}_{5 / 3} \mathrm{Sr}_{1 / 3} \mathrm{NiO}_{4}$ and $\mathrm{La}_{3 / 2} \mathrm{Sr}_{1 / 2} \mathrm{NiO}_{4}$, respectively, was ascribed to "charge glassiness" or polaron hopping. Another appealing possibility is heterogeneity due to spontaneously forming inhomogeneous charge distribution. Such so-called charge order, manifested as stripelike ordering of holes, is a well established phenomenon in the system $\mathrm{La}_{2-x} \mathrm{Sr}_{x} \mathrm{NiO}_{4}$ (e.g., Ref. 12). An enhanced dielectric constant due to charge-order-induced heterogeneity still represents a kind of MW effect. However, within this framework, the origin of the MW relaxation leading to the apparently colossal $\varepsilon^{\prime}$ is inherent to the material, and thus this effect can be regarded as quasi-intrinsic. In addition, in this case, the heterogeneity arises on a much finer scale than, e.g., for contact or grain boundary effects, which may be advantageous for application in highly miniaturized devices.
In summary, a detailed dielectric investigation of a charge ordered nickelate up to $1 \mathrm{GHz}$ has revealed colossal values of the dielectric constant, retaining their colossal magnitude well into the technically relevant gigahertz frequency region. The dielectric properties of LSNO are superior to those of other colossal $\varepsilon^{\prime}$ materials, including the intensely investigated CCTO. Our measurements with different contact types demonstrate that surface effects significantly contribute to the colossal $\varepsilon^{\prime}$. In addition, also charge ordering may play an important role in the generation of the observed unusual dielectric properties. Having in mind that, despite strong efforts, even for the much investigated CCTO no consensus has been achieved so far, it is obvious that final clarification needs further work. In any case, it is clear that here we have a material that deserves at least as much attention as CCTO, both from an application and an academic point of view.

This work was supported by the Deutsche Forschungsgemeinschaft via the Sonderforschungsbereich 484 and by the Commission of the European Communities, STREP: NUOTO, Grant No. NMP3-CT-2006-032644. Ruud Hendrikx at the Department of Materials Science and Engineering of the Delft University of Technology is acknowledged for the X-ray analysis of the sample.

${ }^{1}$ M. A. Subramanian, D. Li, N. Duan, B. A. Reisner, and A. W. Sleight, J. Solid State Chem. 151, 323 (2000).

${ }^{2}$ C. C. Homes, T. Vogt, S. M. Shapiro, S. Wakimoto, and A. P. Ramirez, Science 293, 673 (2001).

${ }^{3}$ C. M. Rey, H. Mathias, L. R. Testardi, and S. Skirius, Phys. Rev. B 45, 10639 (1992); J. L. Cohn, M. Peterca, and J. J. Neumeier, ibid. 70, 214433 (2004); J. Rivas, B. Rivas-Murias, A. Fondado, J. Mira, and M. A. Senaris-Rodriguez, Appl. Phys. Lett. 85, 6224 (2004).

${ }^{4}$ T. Park, Z. Nussinov, K. R. A. Hazzard, V. A. Sidorov, A. V. Balatsky, J. L. Sarrao, S.-W. Cheong, M. F. Hundley, J.-S. Lee, Q. X. Jia, and J. D. Thompson, Phys. Rev. Lett. 94, 017002 (2005).

${ }^{5}$ X. Q. Liu, S. Y. Wu, X. M. Chen, and H. Y. Zhu, J. Appl. Phys. 104, 054114 (2008).

${ }^{6}$ P. Lunkenheimer, R. Fichtl, S. G. Ebbinghaus, and A. Loidl, Phys. Rev. B 70, 172102 (2004).

${ }^{7}$ D. C. Sinclair, T. B. Adams, F. D. Morrison, and A. R. West, Appl. Phys. Lett. 80, 2153 (2002).

${ }^{8}$ P. Lunkenheimer, V. Bobnar, A. V. Pronin, A. I. Ritus, A. A. Volkov, and A. Loidl, Phys. Rev. B 66, 052105 (2002).

${ }^{9}$ S. Krohns, P. Lunkenheimer, S. G. Ebbinghaus, and A. Loidl, Appl. Phys. Lett. 91, 022910 (2007).

${ }^{10}$ S. Krohns, P. Lunkenheimer, S. G. Ebbinghaus, and A. Loidl, J. Appl Phys. 103, 084107 (2008).

${ }^{11}$ C. H. Chen, S. W. Cheong, and A. S. Cooper, Phys. Rev. Lett. 71, 2461 (1993).

${ }^{12}$ V. Sachan, D. J. Buttrey, J. M. Tranquada, J. E. Lorenzo, and G. Shirane, Phys. Rev. B 51, 12742 (1995); J. M. Tranquada, D. J. Buttrey, and V. Sachan, ibid. 54, 12318 (1996).

${ }^{13}$ U. Schneider, P. Lunkenheimer, A. Pimenov, R. Brand, and A. Loidl, Ferroelectrics 249, 89 (2001).

${ }^{14}$ See EPAPS Document No. E-APPLAB-94-071912 for supplementary details. For more information on EPAPS, see http://www.aip.org/pubservs/ epaps.html.

${ }^{15}$ J. L. Zhang, P. Zheng, C. L. Wang, M. L. Zhao, J. C. Li, and J. F. Wang, Appl. Phys. Lett. 87, 142901 (2005).

${ }^{16}$ A. K. Jonscher, Nature (London) 267, 673 (1977).

${ }^{17}$ S. R. Elliott, Adv. Phys. 36, 135 (1987).

${ }^{18}$ Ch. Kant, T. Rudolf, F. Mayr, S. Krohns, P. Lunkenheimer, S. G. Ebbinghaus, and A. Loidl, Phys. Rev. B 77, 045131 (2008). 